\title{
Factores asociados a la deserción de estudiantes en la carrera virtual de Abogacía de una universidad pública
}

Factors associated to desertion of students of the virtual career of Advocacy in a public university

Fatores associados à evasão de alunos da carreira virtual de Direito em uma universidade pública

\author{
Martín Ritorni Baz \\ Universidad Nacional del Chaco Austral \\ Departamento de Ciencias Sociales, Chaco, Argentina \\ martinritornibaz@gmail.com \\ https://orcid.org/0000-0001-7954-450X
}

Gisela Paola Gómez
Universidad Nacional del Chaco Austral
Departamento de Ciencias Sociales. Chaco, Argentina
giselagomez@ uncaus.edu.ar
https://orcid.org/0000-0002-4407-8921

Germán Eduardo Oestmann

Universidad Nacional del Chaco Austral Departamento de Ciencias Sociales, Chaco, Argentina germanoestmann@uncaus.edu.ar https://orcid.org/0000-0003-3820-1951

\author{
Nancy Liliana Salin \\ Universidad Nacional del Chaco Austral \\ Departamento de Ciencias Sociales, Chaco, Argentina \\ nancysalin@uncaus.edu.ar \\ https://orcid.org/0000-0002-9060-6254 \\ Mara Cristina Romero \\ Universidad Nacional del Chaco Austral \\ Departamento de Ciencias Básicas y Aplicadas \\ Chaco, Argentina \\ mara@uncaus.edu.ar \\ https://orcid.org/0000-0001-9624-9051
}

\begin{abstract}
RESUMEN
El propósito de este trabajo fue determinar las causas que llevan al estudiante a abandonar la carrera virtual de Abogacía de la Universidad Nacional del Chaco Austral. Para esto se realizó un estudio exploratorio - descriptivo y se aplicó una encuesta de elaboración propia, mediante la herramienta Google Forms, a todos los alumnos que se encuentran cursando la carrera, al finalizar el primer cuatrimestre de 2019. Se obtuvieron un total de $n=4911$ de respuestas, que mostraron que más del 50\% de los estudiantes de Abogacía residen a más de $200 \mathrm{Km}$ de distancia de la Universidad, poseen entre 30 y 39 años y trabajan a tiempo parcial o completo. Factores como falta de tiempo para estudiar y presentar trabajos, dificultan el cursado de la carrera. Además, la falta de comunicación entre alumnos y docentes lleva a la frustración de los mismos, siendo la principal causa de abandono, demostrando la necesidad de implementar herramientas pedagógicas para subsanar la comunicación. Con los resultados obtenidos se espera, ampliar y mejorar el curso de ingreso a la universidad, permitirá al ingresante contar con los elementos necesarios para llevar adelante la carrera.
\end{abstract}

Palabras clave / Descriptores: Abandono de estudio. Autoaprendizaje. Calidad de la educación. Cuestionario online. Enseñanza a distancia.

\section{ABSTRACT}

The aim of this work was to determine the causes that lead the student to abandon the virtual career of Law at the National University of Chaco Austral. For this, an exploratory-descriptive study was carried out and a self-elaborated survey was applied, using the Google Forms tool, to all students who are studying the degree, at the end of the first semester of 2019. A total of $\mathrm{n}=4,911$ responses, which showed that more than $50 \%$ of Law students reside more than $200 \mathrm{~km}$ away from the University, are between 30 and 39 years old and work part or full time. Factors such as lack of 
time to study and present work make it difficult to complete the degree. In addition, the lack of communication between students and teachers leads to their frustration, being the main cause of abandonment, demonstrating the need to implement pedagogical tools to correct communication. With the results obtained, it is expected, expanding and improving the university entrance course, will allow the entrant to have the necessary elements to carry out the career.

Keywords: Abandonment of study. Self-learning. Quality of education. Online questionnaire. Teachings to distance.

\section{RESUMO}

O objetivo deste trabalho foi determinar as causas que levam o estudante a abandonar a carreira virtual de Direito na Universidad Nacional del Chaco Austral. Para tal, foi realizado um estudo exploratório-descritivo e aplicado um enquete, utilizando a ferramenta Google Forms, a todos os alunos que estão a frequentar a licenciatura, no final do primeiro trimestre de 2019. Um total de $n=4911$ respostas, o que mostrou que mais de $50 \%$ dos estudantes de Direito residem a mais de $200 \mathrm{~km}$ da Universidade, têm entre 30 e 39 anos e trabalham meio período ou período integral. Fatores como falta de tempo para estudar e apresentar trabalhos dificultam a conclusão do curso. Além disso, a falta de comunicação entre alunos e professores leva à sua frustração, sendo a principal causa de abandono, demonstrando a necessidade de implementar ferramentas pedagógicas para uma comunicação correta. Com os resultados obtidos, espera-se ampliar e aprimorar o curso de ingresso na universidade, o que permitirá ao candidato ter os elementos necessários para realizar a carreira.

Palavras-chave: Abandono do estudo; Auto-aprendizagemç Qualidade da educação; Questionário on-line. Ensino à distância.

\section{INTRODUCCIÓN}

La educación universitaria en entornos virtuales presenta en nuestro país un sostenido crecimiento, contando con factores positivos o negativos según cómo el alumno los utilice, como la dedicación horaria, autogestión de los tiempos de estudio, localización de los centros de estudios, entre otros. La situación económica, laboral y familiar suelen ser determinantes de la elección de las carreras virtuales, creando grandes expectativas en los alumnos sobre los cursos que se manejan con tecnologías de información, generando un conjunto de motivaciones acerca de esta modalidad. Sin embargo, muchas veces estos factores de índole personal profundizan las limitaciones para el desarrollo del proyecto educativo personal de los estudiantes.

La deserción es el abandono que hace el estudiante de una o varias asignaturas a las que se ha inscripto, cesando su educación en la institución durante el primer año desde la inscripción, es decir no cursando materias durante dos semestres consecutivos (Sneyers \& De Witte, 2017). MEN, (2009) señala que la deserción se puede producir antes de que el estudiante comience el cursado, durante los primeros años de la carrera, o bien en instancias finales de la carrera; siendo además sensible al programa académico empleado por la universidad. Distintos autores abordaron las causas de deserción, encontrando algunas de las variables que afectan al proceso de enseñanza aprendizaje en $\mathrm{EaD}$, entre las que se incluyen la integración social y el compromiso institucional e individual del estudiante con su lugar de estudios, la capacidad intelectual de los mismos, los factores socio-económicos, educativos y demográficos, los aspectos socioculturales y políticos del medio, la influencia familiar, la edad, carencia de procesos afectivos en el proceso de enseñanzaaprendizaje, insuficiente dominio técnico en el uso de la computadora, entre otros.

Durante los últimos años, se observó un cambio en la educación debido a la incorporación de las Tecnologías de Información y Comunicación (TICs) y el uso de plataformas de aprendizaje con el objetivo no solo de mejorar la calidad educativa, sino de facilitar el acercamiento y acceso, de carreras universitarias de grado y posgrado virtuales. La educación universitaria a distancia surge como un moderador frente a la desigualdad de oportunidades, presentándose como una oportunidad de estudios universitarios en los casos de abandono de las modalidades universitarias tradicionales. Sin embargo, aunque esta forma de estudio busca brindar igualdad de oportunidades, la elección de la carrera y la complejidad de los temarios dados a los alumnos exigen un grado distinto de conocimiento que en ocasiones se vuelve insuficiente y poco práctico para el aprendizaje. Por lo que, las universidades que brindan esta modalidad de educación deben conocer las características 
propias del aprendizaje y elaborar estrategias que logren mejorar el nivel de estudio, evitando así la deserción de los discentes.

Este artículo se elaboró como resultado de un proyecto que buscaba identificar cuáles son los factores que llevan al abandono de la carrera virtual, conocer las circunstancias secundarias que inciden en el fenómeno y proponer acciones para evitar el abandono. Los resultados del proyecto permitirán conocer las causas que determinan el abandono de la matrícula en abogacía, lo que puede resultar útil si se evidencian dentro de la investigación elementos que se pueden mejorar dentro del proceso de aprendizaje, para procurar la permanencia del estudiante en el programa educativo.

\section{FUNDAMENTO TEÓRICO}

Actualmente el aprendizaje en entornos virtuales sitúa a los individuos como autogestores de conocimientos, evidenciando su necesidad de intercambiar y socializar con sus pares y docentes interaccionando en una plataforma en un contexto específico de enseñanza-aprendizaje. Si bien la modalidad a distancia presenta algunas ventajas como accesibilidad, costos, flexibilidad horaria y eficacia en la entrega de materiales educativos, entre otros, muchos estudiantes por diferentes motivos que pertenecen o no al ámbito pedagógico abandonan estos cursos (Urbina Cárdenas y Ovalles Rodriguez, 2016). La decisión del estudiante de iniciar o terminar una carrera a distancia se ve afectada por diversos factores como su actitud y adaptación a nuevas metodologías de enseñanza-aprendizaje que demandan compromiso y eficiencia en la comunicación (Hernández, et al., 2018).

Hernández et al., (2014) indican que los nacidos en los 90's, es decir en la era pre digital, en ocasiones se muestran reacios a integrar tecnologías de la comunicación al proceso de enseñanza; en cambio los nativos digitales, están más abiertos a su uso en el aula. Aunque es sabido que existe una brecha académica entre lo que se enseña en las escuelas o preparatorias y lo que se exige en las universidades, egresando de las primeras con bajo nivel de preparación, insuficiencia de formación y deficiencias de conocimientos en general (Viale, 2014; La Madriz, 2016; Arias-Gómez y Aponte, 2017). En este sentido las limitaciones u obstáculos que todavía no están resueltos o no son de conocimiento por los alumnos, como la carencia de habilidades en manejo de herramientas informáticas, la dificultad para el acceso a materiales por equipamiento informático inapropiado o la conexión eficiente a Internet, entre otros favorece el abandono de la carrera.

La deserción es el abandono que hace el estudiante de una o varias asignaturas a las que se ha inscripto, cesando su educación en la institución durante el primer año desde la inscripción, es decir no cursando materias durante dos semestres consecutivos (Sneyers y De Witte, 2017). El Ministerio de Educación Nacional de Colombia, (2009) señala que la deserción se puede producir antes de que el estudiante comience el cursado, durante los primeros años de la carrera, o bien en instancias finales de la carrera. Vázquez y Rodríguez (2007) que establecieron algunas de las variables que afectan al proceso de enseñanza - aprendizaje en Educación a Distancia [EaD], entre las que se incluyen la integración social y el compromiso institucional e individual del estudiante con su lugar de estudios, la capacidad intelectual (relacionada a la capacidad de adaptación y la solución de problemas frente a los desafíos académicos), los factores socio-económicos, educativos y demográficos, el nivel de estudios al que se aspira, los aspectos socioculturales y políticos del medio, la influencia familiar, la edad, entre otros. Asimismo, Fernández Sánchez, (2009) indicaron que algunas de las posibles causas de la deserción de los alumnos de EaD son: sentir abandono por parte de los docentes cuando hay respuesta tardía a sus correos, sentir poco profesionalismo en la revisión de sus trabajos, no admitir otras opiniones o correcciones, no haber desarrollado completamente o correctamente habilidades de lecto comprensión de textos y no respetar su papel de alumnos, entre otras. Estévez, et al., (2015) y Soltero et al., (2014) encontraron, además de las variables mencionadas anteriormente en $\mathrm{EaD}$ la falta de empatía con docentes y pares en el proceso de enseñanza-aprendizaje, desconocimiento de herramientas y aplicaciones empleadas en las plataformas universitarias, lo que dificulta la resolución de las actividades propuestas en los cursos. 
Para prevenir o reducir los abandonos evitables la institución debe tener conocimiento de las causas o factores de deserción, de manera de poder aplicar acciones tendientes a la contención de los estudiantes inscriptos a las carreras virtuales. Así, en base a datos extraídos de seguimientos de inscripciones, recusantes y nuevos ingresos dentro de la Universidad Nacional del Chaco Austral [UNCAUS] en la Carrera de Abogacía, notamos que el 36\% de los alumnos abandonaron el cursado de la carrera en 2018, o bien muestran una lentificación mayor a la media. El presente trabajo se enmarca en el Proyecto de Investigación (PI105/19) denominado: Factores asociados a la deserción en educación a distancia en los primeros años de la carrera de Abogacía de la Universidad Nacional del Chaco Austral. Por lo tanto, el objetivo principal de este trabajo fue determinar las posibles causas que llevan al estudiante a abandonar la carrera virtual de Abogacía de la UNCAUS, de manera que puedan implementarse estrategias de mejoras necesarias para minimizar el porcentaje de abandono, maximizar recursos, mejorar factores endógenos, y contener al alumno durante su cursado.

\section{PROCEDIMIENTOS METODOLOGICOS}

García y Cabero (2011) señalan que el cuestionario es un instrumento tradicional utilizado investigaciones que abordan una diversidad de problemáticas, principalmente en educación. Para la confección del instrumento de recolección de datos se utilizó el modelo de encuesta mixta, mayormente con respuesta preformateadas de tipo opción múltiple (múltiple choice). El diseño de la encuesta comenzó con la lectura de bibliografía que aborda estudios similares, y continuó con el diálogo con docentes de otras carreras virtuales sobre la problemática, a fin de esbozar preguntas que busquen responder a dicha situación.

Debido a que el instrumento fue de elaboración propia, se realizó su validación de contenido mediante juicio de cinco expertos según lo propuesto por (García y Cabero, 2011). De acuerdo a Escofet et al., (2016), algunos de los criterios de selección de los expertos fueron que tuvieran experiencia laboral en el ámbito de la tecnología educativa, o en el ámbito de la formación virtual, e-learning, tener experiencia en formación virtual y entornos personales de aprendizaje, tener una experiencia formativa superior a diez años, pertenecer a diferentes carreras, y haber colaborado anteriormente en otros trabajos de investigación o de publicación. Las respuestas y sugerencias de los jurados fueron procesadas, analizadas y consideradas para la construcción de la versión final de un cuestionario útil para su propósito, según lo propuesto por García y Cabero, (2011).

El cuestionario final se sometió nuevamente a evaluación, encontrándose correcto en consistencia, coherencia y fiabilidad (Hernández y Barrera, 2018). Así, la encuesta final constaba de cuatro bloques: el primero referido a los datos sociodemográficos, con sus variables sexo, edad, situación laboral y distancia de su residencia a la sede de estudio; el segundo a las motivaciones para estudiar esta carrera en la universidad, con sus variables como motivación para elegir la carrera, condición económica; el tercero a educación a distancia con variables como conocimiento en TICs, procesadores de texto; y el cuarto referido al cursado de las materias propias de la carrera.

\subsection{Procedimiento de recogida y análisis de datos}

Se realizó un estudio exploratorio - descriptivo, mediante el análisis de frecuencias y porcentajes de respuesta a las distintas opciones. La encuesta se llevó adelante a través de la plataforma Google empleando la herramienta Google Forms, al finalizar el primer cuatrimestre de 2019. La misma se contestó a través de un link que se proporcionó al momento de su aplicación, de manera que el estudiante era direccionado a una nueva página donde respondía la encuesta, manteniendo así el anonimato (Leyva et al., 2018). Dado que buscamos indagar cuales son las posibles causas de abandono, la encuesta fue aplicada a todos los alumnos que se encuentran cursando la carrera ( $\mathrm{n}=4911$ estudiantes). Las respuestas recolectadas, se obtuvieron en formato de hoja de cálculo de Microsoft Excel 2007. Se realizó un análisis estadístico descriptivo expresando 
porcentaje (\%) y frecuencias (n), para presentar los resultados analizados en base a los contenidos teóricos en tablas y gráficos.

\section{RESULTADOS Y DISCUSIÓN}

Escanés et al., (2014) abordaron las causas de deserción, encontrando algunas de las variables que afectan al proceso de enseñanza - aprendizaje en $\mathrm{EaD}$, entre las que se incluyen la integración social y el compromiso institucional e individual del estudiante con su lugar de estudios, la capacidad intelectual de los mismos, los factores socio-económicos, educativos y demográficos, los aspectos socioculturales y políticos del medio, la influencia familiar, la edad, carencia de procesos afectivos en el proceso de enseñanza-aprendizaje, insuficiente dominio técnico en el uso de la computadora, entre otros. De acuerdo con Tomás y Gutiérrez (2019) el clima motivacional del aula y la satisfacción de las necesidades psicológicas básicas influyen directamente en la satisfacción académica de los estudiantes, lo que se traduce en los entornos virtuales a actividades innovadoras por parte de los docentes y respuestas rápidas en foros ante las consultas de los alumnos, así como a la adecuada autogestión y manejo del tiempo del estudiante para el cumplimento de actividades. Por lo tanto, el conocimiento de las causas o factores de deserción, así como las características de la población estudiada, pueden son el insumo inicial para prevenir o reducir los abandonos evitables en las instituciones educativas.

La invitación a responder el cuestionario obtuvo un total de 4911 respuestas completas, estando el alumnado de la carrera compuesto por $40,9 \%$ hombres y $50,1 \%$ mujeres. Los resultados arrojados en el bloque sociodemográfico se observan en la Tabla 1. Estos datos sirven para caracterizar a la población de estudio, evidenciando que entre los alumnos de abogacía predominan las estudiantes del sexo femenino, permitiéndonos además identificar la mayor cantidad de personas por edades que matriculan en la carrera. Respecto del cursado de la carrera el $69 \%$ de los estudiantes se encuentra cursando los tres primeros años de la carrera como se indica: 40,9\% en primer año, $25,1 \%$ en segundo, $23,1 \%$ en tercero; estando el resto de los alumnos un $8,6 \%$ en cuarto y un $2,3 \%$ en quinto año respectivamente. Evidenciando que factores de índole personal generalmente son determinantes de la elección de las carreras virtuales. La información obtenida respecto del lugar de residencia del alumnado, da indicios sobre porque la mayoría de los estudiantes encuentran en la modalidad virtual la posibilidad de acceder a la educación superior, ya que más del $72 \%$ de los estudiantes reside a una distancia mayor a $100 \mathrm{~km}$ de la universidad. Respecto de la caracterización de la muestra, la misma estuvo compuesta principalmente por mujeres. Otros investigadores como Humanante-Ramos et al., (2019) y Leyva et al., (2018) también encontraron que la mayoría de los inscriptos a cursos virtuales son mujeres, coincidiendo la distribución de edades del alumnado con lo informado por Bermúdez et al., (2016). Asimismo, más del $72 \%$ de los estudiantes reside a una distancia mayor a $100 \mathrm{~km}$ de la universidad, lo que explica además la elección de la modalidad de cursado. Busso y Perez (2015) y muestran que la dificultad de combinar estudios y trabajo afecta en general más a jóvenes. En nuestro estudio podemos inferir, por la edad de los estudiantes que éstos pueden compaginar estudios y trabajo, residir en domicilio propio y muy probablemente tienen cargas familiares, y aunque se inscriban a estas carreras sin tener demasiado claras las exigencias que implicaba una carrera de grado (Ministerio Nacional de Educación, 2009).

Entre las distintas variables estudiadas, la situación económica, laboral y familiar suelen ser determinantes de la elección de las carreras virtuales, creando grandes expectativas en el alumnado y su entorno con respecto a la modalidad. Sin embargo, muchas veces estos factores de índole personal profundizan las limitaciones para el desarrollo del proyecto educativo personal de los estudiantes. Lo encontrado en nuestra encuesta, respecto de estas preguntas coincide parcialmente con lo informado por Mano et al., (2013), quienes hallaron en su estudio que los estudiantes consideran la titulación como una oportunidad de superación personal y para demostrar sus capacidades (desarrollo profesional) y en función de la percepción social de la profesión (ser útil a la sociedad). Además, la gratuidad de la carrera, es un factor que no se ha encontrado en estudios 
similares e influye en la elección de la misma ya que los alumnos consideran que el factor económico es importante en la selección de la carrera. Se pudo observar que la vocación por el estudio y ejercicio del derecho figura en último lugar por los cuales se eligieron la carrera. El derecho, tanto en enseñanzas occidentales como anglosajonas presentan constantemente terminologías propias y conceptualizaciones en abstracto. El cursante de la carrera presenta falencias de vocabulario, comprensión de textos e interpretación de consignas, aunque consideren que el lenguaje se no es una dificultad al estudiar. Este asunto genera complicaciones tanto para el alumno mismo como para los docentes que se ven en la necesidad de no solamente enseñar la materia, sino también realizar explicaciones excesivas sobre la realización de trabajos. A la hora de los exámenes esto genera disgustos en el alumnado que se frustra y se molesta con la modalidad. Esto pudo observarse en los campos de completado manual en la encuesta en cuestión.

El estudiante de la carrera de abogacía debe enfrentarse a una abundante parte de construcción teórica del derecho, una parte práctica, manejo de la oralidad, análisis de casos y filosofía, solo por citar algunos. Respecto de las motivaciones del alumnado para la elección de la carrera universitaria, la Figura 1 muestra los principales motivos de la elección de la carrera Abogacía en la UNCAUS, presentándose en gris aquellos motivos de índole personal y en negro los referidos a la casa de altos estudios. Estos factores influyen en el logro de determinadas metas académicas y personales, estando a su vez afectados por un grupo de factores que a su vez se interrelacionan entre sí y que son propios de las características socioeconómicas y demográficas del alumno (González, 2005). Al indagar los motivos por los cuales se decide iniciar la carrera de derecho, figura la gratuidad en primer lugar (79\%), seguido por el desarrollo profesional y gratuidad de la matrícula fueron las opciones más seleccionadas por los estudiantes. Cabe destacar que en los entornos presenciales demanda un gran esfuerzo económico para solventar gastos de mantención, más aún si tenemos en cuenta que los estudiantes trabajan a tiempo completo.

Tabla 1. Características sociodemográficas de los Alumnos de Abogacía Virtual de la UNCAUS

\begin{tabular}{lcc}
\hline Edad de los alumnos (años) & $\mathrm{n}$ & $\mathrm{F} \%)$ \\
\hline Menos de 21 & 287 & 5,9 \\
de 21 a 29 & 1321 & 26,9 \\
de 30 a 39 & 1801 & 36,7 \\
de 40 a 50 & 1097 & 22,3 \\
más de 50 & 405 & 8,2 \\
\hline Lugar de residencia respecto a la sede de la universidad & \\
\hline Distancia (Km) & $\mathrm{n}$ & $\mathrm{F}(\%)$ \\
Reside en la ciudad de la sede & 717 & 14,6 \\
Reside hasta 100 Km de la sede & 629 & 12,8 \\
Reside entre 100 a 200 Km & 1547 & 31,5 \\
Reside a más de 200 Km & 2018 & 41,1 \\
\hline Situación laboral & $\mathrm{n}$ & $\mathrm{F}(\%)$ \\
\hline Ni trabajo ni busco trabajar & 172 & 3,5 \\
Estoy buscando trabajo & 727 & 14,8 \\
Trabajo a tiempo parcial & 1606 & 32,7 \\
Trabajo a tiempo completo & 2406 & 49 \\
\hline
\end{tabular}

Nota: Elaboración propia

Figura 1. Motivos de selección de la carrera 


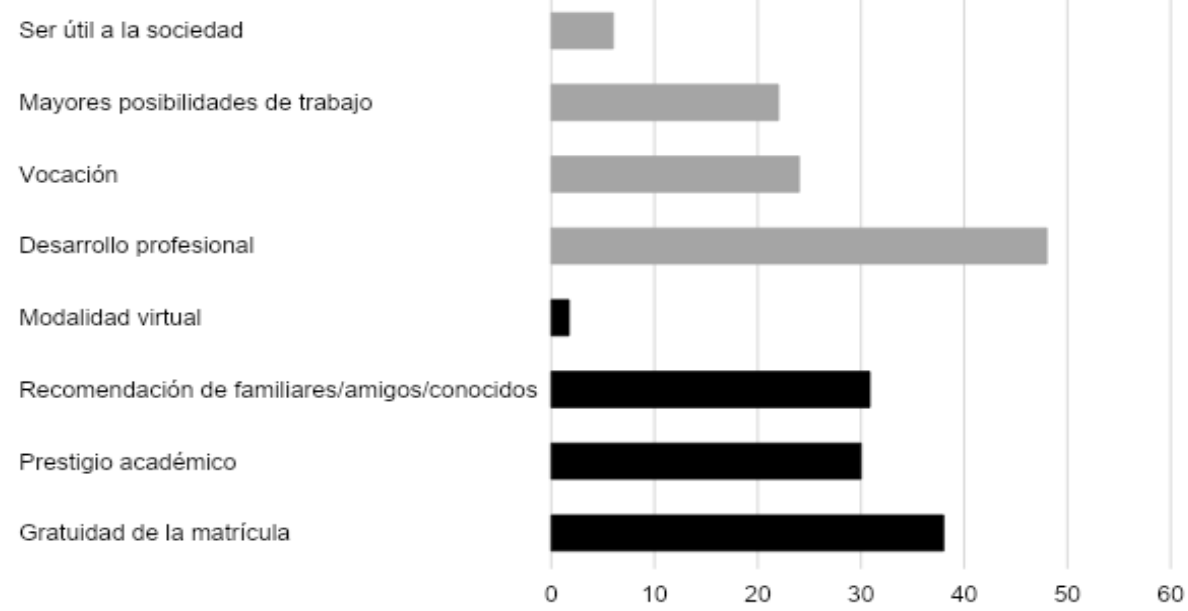

Nota: elaboración propia.

La Tabla 2 resume algunos aspectos referidos a las habilidades de los alumnos sobre manejo de TICS. En los entornos virtuales el manejo de procesadores de texto, soportes audiovisuales e internet son importantes ya que podrían predisponer a la deserción de la carrera por el manejo tecnológico. Sin embargo, se puede observar en la Tabla 2 que aproximadamente el $50 \%$ de los encuestados poseen conocimientos básicos de esas herramientas y el $43 \%$ manejo básico de internet y videoconferencias. Además, solo el $32 \%$ de los alumnos indicaron haber realizado y finalizado un curso virtual, aunque consideran de manejo normal o fácil la plataforma empleada por la universidad para el dictado de sus materias.

Tabla 2. Conocimientos de los alumnos sobre manejo de herramientas informáticas

\begin{tabular}{lcc}
\hline $\begin{array}{l}\text { Conocimientos de procesador de texto } \\
\text { (Ej. MS Word) }\end{array}$ & $\mathrm{n}$ & $\mathrm{F}(\%)$ \\
\hline Nulos & 657 & 14 \\
Básicos & 2381 & 48 \\
Avanzados & 1873 & 38 \\
\hline $\begin{array}{l}\text { Conocimientos de planilla de cálculo } \\
\text { (Ej. MS Excel) }\end{array}$ & $\mathrm{n}$ & $\mathrm{F}(\%)$ \\
\hline Nulos & 909 & 18 \\
Básicos & 2544 & 52 \\
Avanzados & 1458 & 30 \\
\hline Conocimientos de soporte audiovisual & $\mathrm{n}$ & $\mathrm{F}(\%)$ \\
(Ej. MS PowerPoint) & 763 & 16 \\
\hline Nulos & 2525 & 51 \\
Básicos & 1623 & 33 \\
Avanzados & $\mathrm{n}$ & $\mathrm{F}(\%)$ \\
\hline Manejo de internet & 602 & 12 \\
\hline Nulos & 2116 & 43 \\
$\begin{array}{l}\text { Básicos } \\
\text { Avanzados }\end{array}$ & 2193 & 45 \\
\hline Manejo de videoconferencias & $\mathrm{n}$ & $\mathrm{F} \%)$ \\
con relación a la modalidad virtual & 2960 & 60 \\
\hline Es la primera vez que realiza un & 1554 & 32 \\
curso/carrera virtual & 381 & 8 \\
Realizó y no finalizó cursos virtuales & $\mathrm{n}$ & $\mathrm{F} \%)$ \\
Realizó y finalizó cursos virtuales & & \\
\hline Considera al manejo de la plataforma \\
virtual de la UNCAUS
\end{tabular}




\begin{tabular}{lcc}
\hline Muy difícil & 57 & 1 \\
Difícil & 285 & 6 \\
Normal & 3291 & 67 \\
Fácil & 905 & 18 \\
Muy fácil & 373 & 8 \\
\hline
\end{tabular}

Nota: elaboración propia.

Los conocimientos previos que posee el alumnado al ingresar a la universidad facilitan o dificultan su paso por ésta. Hernández et al., (2018) encontraron que un conocimiento y manejo previos de herramientas de internet influyen positivamente en la actitud de los estudiantes hacia la educación en línea. Esto concuerda con los resultados obtenidos en nuestra investigación, donde se encontró que los alumnos poseen conocimientos básicos de procesadores de texto, soportes audiovisuales e internet. Además, estos resultados coinciden parcialmente con lo informado por Soto, et al., (2015) y Leyva (2018) quienes encontraron que las personas prefieren realizar cursos virtuales que presenciales, lo que podría atribuirse a la autogestión del tiempo y a las habilidades personales de los alumnos con respecto al uso de las TIC para afrontar estudios a distancia. Asimismo, el estudio realizado por Marín et al., (2017) demuestra que en general los alumnos consideran a las plataformas de educación virtual accesible y fácil de emplear, permitiendo el acceso a ellas desde cualquier lugar y en cualquier momento. Humanante-Ramos et al., (2019) señalan que los contenidos publicados en los entornos virtuales, la organización y la claridad al dar la clase, se evidencia en las actividades de trabajo planteadas por los docentes que motivan la generación de nuevos aprendizajes, evaluando positivamente esta forma de aprendizaje, lo que coincide con lo informado por Duque et al., (2013). Asimismo, Peralta y Mora (2016) informaron que los estudiantes de entornos virtuales manifestaron que su abandono obedecía principalmente a la dificultad con las asignaturas, en cuanto a actividades y manejo de tiempo para la realización de tareas.

En cuanto al material de clases, elaborado por los docentes, los alumnos lo consideran adecuado, señalando además que profundizan los saberes que consideran necesarios con la bibliografía sugerida por los docentes, lo cual se puede observar en la Tabla 3. Al ser una carrera virtual, y dado que aproximadamente el 50\% del alumnado trabaja, el tiempo para estudiar, es decir, la autogestión del tiempo para dedicarle a las materias es uno de los mayores inconvenientes encontrados durante la cursada, por lo que puede entenderse cuando los alumnos señalan que solo emplean el material dado por los docentes para estudiar y no la bibliografía sugerida.

Tabla 3. Calificación dada por los alumnos al material elaborado/sugerido por los docentes

\begin{tabular}{lcc}
\hline Material elaborado por los docentes & $\mathrm{n}$ & $\mathrm{F}(\%)$ \\
\hline Muy escaso & 71 & 1 \\
Escaso & 405 & 8 \\
Adecuado & 4267 & 87 \\
Excesivo & 168 & 3 \\
\hline Bibliografía manejada/sugerida durante el cursado & $\mathrm{n}$ & $\mathrm{F}(\%)$ \\
\hline Solo utilizo el material didáctico otorgado por las & 763 & 16 \\
docentes & 2525 & 51 \\
Siempre utilizo bibliografía sugerida por las docentes & 1623 & 33 \\
No utilizo bibliografía sugerida por las docentes & \\
\hline
\end{tabular}

Nota: Elaboración propia

La Tabla 4 resume algunos aspectos referidos a la cursada de la carrera. La forma de presentación de las actividades mantiene al alumnado interesado por los nuevos saberes, sin embargo, solo el $6 \%$ del alumnado considera que las actividades de los profesores son innovadoras, 
lo que podría influir en la motivación, e interés de los mismos durante la cursada; aunque las consignas de las mismas se entiendan, y lo solicitado en las actividades sea adecuado para el tiempo de presentación. Además, el 52\% señalan que los docentes no responden sus preguntas en los foros, lo que dificulta la correcta interpretación y fijación de los contenidos dados.

Respecto del lenguaje específico, el manejado por el cuerpo docente es comprensible, aunque al ser técnico jurídico, no siempre se espera que esto suceda. La elaboración de material por parte de los docentes facilita el entendimiento del lenguaje propio de la carrera.

Tabla 4. Aspectos referidos al cursado de materias en la carrera Abogacía respecto al material entregado por los docentes

\begin{tabular}{|c|c|c|}
\hline $\begin{array}{l}\text { Presentan actividades innovadoras que promueven } \\
\text { la motivación }\end{array}$ & $\mathrm{n}$ & $\mathrm{F}(\%)$ \\
\hline Siempre & 302 & 6 \\
\hline A veces & 2670 & 54 \\
\hline Nunca & 1939 & 40 \\
\hline Comprende el enunciado de las consignas & $\mathrm{n}$ & $\mathrm{F}(\%)$ \\
\hline Siempre & 1912 & 39 \\
\hline A veces & 2950 & 60 \\
\hline Nunca & 49 & 1 \\
\hline $\begin{array}{l}\text { Considera que el tiempo de presentación de } \\
\text { actividades es }\end{array}$ & $\mathrm{n}$ & $\mathrm{F}(\%)$ \\
\hline Escaso & 774 & 16 \\
\hline Adecuado & 3969 & 81 \\
\hline Excesivo & 168 & 3 \\
\hline $\begin{array}{l}\text { Vocabulario técnico jurídico del material didáctico } \\
\text { según año cursado }\end{array}$ & $\mathrm{n}$ & $\mathrm{F}(\%)$ \\
\hline Incomprensible & 72 & 1 \\
\hline Medianamente comprensible & 2397 & 49 \\
\hline Totalmente comprensible & 2442 & 50 \\
\hline $\begin{array}{l}\text { Respuestas de los profesores en los foros para } \\
\text { consulta }\end{array}$ & $\mathrm{n}$ & $\mathrm{F}(\%)$ \\
\hline Siempre & 2703 & 55 \\
\hline A veces & 1873 & 38 \\
\hline Nunca & 335 & 7 \\
\hline $\begin{array}{l}\text { Tiempo tomado por los docentes en responder en } \\
\text { foros }\end{array}$ & $\mathrm{n}$ & $\mathrm{F}(\%)$ \\
\hline Siempre & 3214 & 48 \\
\hline A veces & 1132 & 43 \\
\hline Nunca & 438 & 9 \\
\hline
\end{tabular}

Nota: Elaboración propia

Falta de comunicación con sus pares y docentes y cumplir con los trabajos prácticos son las mayores dificultades encontradas, señalando los alumnos que estos inconvenientes los han llevado a recursar materias, también debido a la falta de devolución de dudas después de los exámenes parciales (Figura 2). Cuando se les preguntó a los alumnos ¿Qué opina de la modalidad de trabajo en grupo?, las respuestas que más se presentan son: me parece útil, siempre y cuando sea por afinidad y en un número reducido, complicado por la cantidad de integrantes del grupo, interesante, por lo que será necesario revisar esta modalidad de trabajo en el futuro.

Figura 2: Dificultades observadas por los alumnos durante el cursado de materias. 


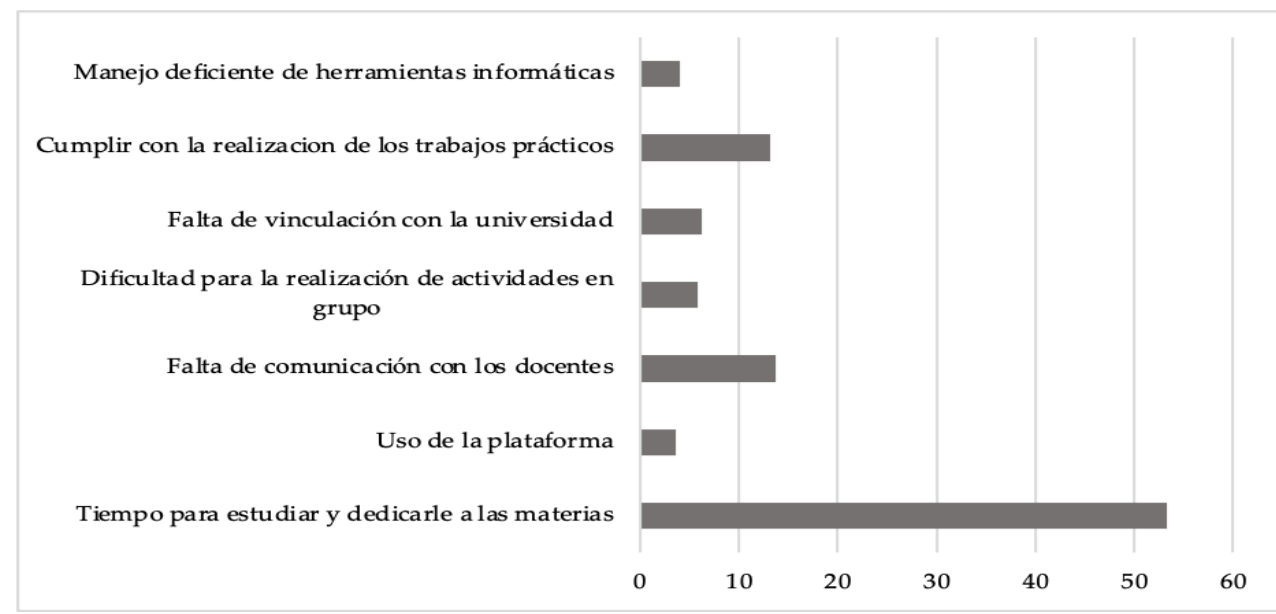

Nota: Elaboración propia

El derecho, tanto en enseñanzas occidentales como anglosajonas presentan constantemente terminologías propias y conceptualizaciones en abstracto. El cursante de la carrera presenta falencias de vocabulario, comprensión de textos e interpretación de consignas. Los análisis lógicojurídicos se acomplejan en una persona que no posee las herramientas de lectura básica y tampoco logran arreglarse con alternativas para la comprensión durante el cursado. Este asunto genera complicaciones tanto para el alumno mismo como para los docentes que se ven en la necesidad de no solamente enseñar la materia, sino también realizar explicaciones excesivas sobre la realización de trabajos. A la hora de los exámenes esto genera disgustos en el alumnado que se frustra y se molesta con la modalidad. Esto pudo observarse en los campos de completado manual en la encuesta en cuestión.

Se debe destacar que en la modalidad a distancia el alumno debe adoptar una conducta de aprendizaje autónomo y de autogestión de aprendizaje, lo que se vislumbra en el manejo del tiempo para presentación de actividades y trabajo en grupo, lo cual es más difícil de realizar debido a la autogestión y organización del tiempo de manera de conciliar horarios y metodologías grupales. En nuestro estudio los alumnos encontraron que el material de clases elaborado por los docentes es adecuado, aunque sus actividades no son innovadoras, siendo el tiempo para estudiar el mayor motivo por el que profundizan poco en la bibliografía dada por los docentes, por lo que interpretamos que puede haber falencias del aprendizaje. Los mayores problemas de los estudiantes al cursar materias se encuentran en la falta de tiempo para realizar sus estudios, seguido de la utilización del tiempo para la realización de trabajos prácticos. Soto et al., (2015) indican que uno de los motivos de la deserción de cursos virtuales es la falta de tiempo para completar tareas. También Lezcano y Vilanova (2017), encontraron que los estudiantes indicaron que es importante la comunicación vía mail, chats y foros de consultas, para equiparar la falta de intercambio presencial. Esto permite inferir que los estudiantes conocen las herramientas de las plataformas, aunque son los docentes quienes deben apoyar e incentivar el uso de las mismas.

\section{CONCLUSIÓN}

Es el primer estudio que se realiza para determinar las posibles causas que llevan al estudiante a abandonar la carrera virtual de Abogacía de la UNCAUS, de manera que puedan implementarse estrategias de mejoras necesarias para minimizar el porcentaje de abandono, maximizar recursos, mejorar factores endógenos, y contener al alumno durante su cursado. En general se necesitan estudios mixtos que permitan trabajar con distintas variables para establecer el grado con el que se puede predecir la deserción en los cursos a distancia a partir de las mismas. Una limitación del estudio podría ser no haber contemplado una fase exploratoria del estudio, contemplando información como apoyo familiar, habilidades informáticas previas, entre otras, través de entrevistas a los estudiantes, habiendo formulado el cuestionario en base a estudios 
similares en otras universidades. La profundización en dichas variables, conjuntamente con financiamiento, motivación interna, cursos previos, satisfacción, deberán ser abordadas en etapas siguientes, para una mejor predicción de las causas de abandono en carreras virtuales.

Sin embargo, como se desprende en el análisis, en nuestro estudio parecería haber una falta de entendimiento entre alumnos y docentes que se desdibujan a causa de la virtualidad y que llevan a la frustración de los mismos al no tener siempre respuesta en tiempos adecuados para el cursado de las distintas materias. La falta de comunicación entre estudiantes es también un impedimento para cumplir con los trabajos prácticos, ya que las dudas no son comunicadas entre compañeros tampoco, lo que puede ocasionar que los alumnos deban recursar asignaturas. Para este asunto, la capacitación pedagógica del cuerpo docente debe considerarse para mejorar el entendimiento de los requerimientos realizados por los docentes a los alumnos. El cuerpo docente debe ser consciente de la importancia de responder a los correos de los estudiantes en tiempo y forma para satisfacer sus dudas y así mejorar su desempeño como estudiante teniendo un impacto positivo en la motivación en el cursado de la asignatura. Favorecer el diálogo entre docentes y alumnos favorecerá el proceso de enseñanza-aprendizaje ya que los mismos estudiantes pueden favorecer la comprensión de temas entre compañeros, siempre que el diálogo sea supervisado por el docente. Además, la carga de respuestas a los alumnos en forma de video para las consultas realizadas, suplantando la clásica respuesta escrita en los foros de trabajo es una manera ágil y clara de dar respuestas. Se observó también un tendencial atractivo del alumnado por la integración en grupos y el interés por la de vinculación con la universidad. Consideramos así que la implementación de planes de extensión universitaria en conjunto grupos de alumnos, podría ser una forma acorde de llegar a saldar esa deuda de presencia e integración; donde a su vez aprovecha la oportunidad de alcanzar a la sociedad a profesionales y futuros profesionales del derecho comprometidos con el medio. Se podría además ampliar y mejorar el curso de ingreso a la universidad, sobre todo, en el manejo de la plataforma Moodle y sus herramientas a fin de que el ingresante pueda contar con los elementos necesarios para llevar adelante la carrera.

Esta investigación tuvo como principio la obtención de datos para establecer planes de mejora basado en estándares de calidad educativa. Habiendo relatado lo anterior, consideramos que surgen los ejes de trabajo a futuro, siendo completamente atendibles y de implementación mediante planificación. Futuros trabajos abordarán sobre la formación académica que reciben los estudiantes de Abogacía, específicamente en las competencias propias de la carrera. En este sentido, la opinión de los egresados sobre la formación profesional recibida ha sido implementada en distintas carreras universitarias de manera de efectuar mejoras o reformas curriculares consideradas necesarias para el profesional. La calidad educativa debe contemplarse de manera integral, abarcando todo el proceso de formación, es decir, desde el ingreso del alumno a la universidad, evidenciando problemáticas iniciales, su recorrido universitario contemplando posibles causas de abandono y por último, los requerimientos del egresado en su inserción laboral, mejorando las competencias de los mismo.

\section{Agradecimientos}

Los autores agradecen al personal de Secretaria Académica y el Departamento de Sistemas de la Universidad Nacional del Chaco Austral quienes colaboraron para que la implementación de la encuesta se realice en forma correcta.

\section{REFERENCIAS}

Arias-Gómez, D. y Aponte, E. (2017). Persistencia académica en un programa de nivelación universitario venezolano: caso Universidad Simón Bolívar. Revista Digital de Investigación en Docencia Universitaria,11, 289-307. https://doi.org/10.19083/ridu.11.512

Bermúdez Rodríguez, F.; Lapaz Castillo, J. L. y Fueyo Gutiérrez, M A. (2016). Percepción y valoración del alumnado sobre las propuestas b-Learning en grados de ingenierías industriales y de Telecomunicación. Didáctica, Innovación y Multimedia (DIM), 34, 1-16. http://dimglobal.net/revistaDIM34/DIMAR34blearning.htm 
Busso, M. y Perez, P. E. (2015). Combinar trabajo y estudios superiores ¿Un privilegio de jóvenes de sectores de altos ingresos? Población \& Sociedad, 22(1), 5-29. http://ppct.caicyt.gov.ar/index.php/pys/article/view/3302/pdf

Cruz Piñeiro, R.; Vargas Valle, E. D.; Hernández Robles, A. K. y Rodríguez Chávez, Ó. (2017). Adolescentes que estudian y trabajan: factores sociodemográficos y contextuales. Revista Mexicana de Sociología, 79(3), 571-604. https://doi.org/10.22201/iis.01882503p.2017.3.57679

Duque, P. A.; Vallejo A., S. L. y Rodríguez R., J. C. (2013). Prácticas pedagógicas y su relación con el desempeño académico [Tesis de Maestría en Educación y Desarrollo Humano]. Universidad de Manizales. Centro de Estudios Avanzados en Niñez y Juventud alianza de la Universidad de Manizales y el CINDE. Manizales. Colombia. http://biblioteca.clacso.edu.ar/Colombia/alianza-cinde-umz/20140805022434/paulaandreaduque.pdf

Escanés, G.; Herrero, V.; Merlino, A. y Ayllón, S. (2014). Deserción en educación a distancia: Factores asociados a la elección de modalidad como desencadenantes del abandono universitario. Virtualidad, Educación y Ciencia, 5(9), 45-55. https://revistas.unc.edu.ar/index.php/vesc/article/view/9549

Escofet, A., Folgueiras, P., Luna, E. y Palou, B. (2016). Elaboración y validación de un cuestionario para la valoración de proyectos de aprendizaje-servicio. RMIE, Revista Mexicana de Investigación Educativa, 21(70), 929-949. http://www.scielo.org.mx/pdf/rmie/v21n70/1405-6666-rmie-21-70-00929.pdf

Estévez, J. A., Castro-Martínez, J. y Rodríguez-Granobles, H. (2015). La educación virtual en Colombia: exposición de modelos de deserción. $\quad$ Apertura, $\quad 7(1), \quad 1-10$. http://www.udgvirtual.udg.mx/apertura/index.php/apertura/article/view/583

Fernández Sánchez, N. (2009). Estilos de aprendizaje entre jóvenes y adultos. Consideraciones andragógicas para la $\begin{array}{lllllll}\text { educación continua } & \mathrm{y} & \mathrm{a} & \text { distancia. } & \text { Revista } & \text { Cognición, } & 17 .\end{array}$ http://www.cognicion.net/index.php?option=com_content\&view=article\&id=216:estilos-de-aprendizaje-entrejvenes-y-adultos- $\&$ catid $=66$ :investigacin $\&$ Itemid $=173$

García, E. y Cabero, J. (2011). Diseño y validación de un cuestionario dirigido a describir la evaluación en procesos de educación a distancia. EDUTEC, Revista Electrónica de Tecnología Educativa, 35 (1). http://edutec.rediris.es/revelec2/revelec35/

Hernández, H. A. y Barrera, A. E. P. (2018). Validación de un instrumento de investigación para el diseño de una metodología de autoevaluación del sistema de gestión ambiental. Revista de Investigación Agraria y Ambiental, 9(1). DOI: https://doi.org/10.22490/21456453.2186

Hernández Godoy, V., Fernández Morales, K. y Pulido, J. (2018). La actitud hacia la educación en línea en estudiantes universitarios. Revista de Investigación Educativa, 36(2), 349-364. https://doi.org/10.6018/rie.36.2.277451. Disponible en: https://revistas.um.es/rie/article/view/277451/231301

Hernández y Hernández, D., Ramírez-Martinell, A. y Cassany, D. (2014). Categorizando a los usuarios de sistemas digitales. Pixel-Bit. Revista de Medios y Educación, 44, 113-126. https://www.redalyc.org/articulo.oa?id=36829340008

Humanante-Ramos, P; Fernández-Acevedo. J y Jiménez, C. (2019). Aulas virtuales en contextos universitarios: percepciones de uso por parte de los estudiantes. Revista Espacios, 40(2), 3-17. https://www.revistaespacios.com/a19v40n02/19400203.html

García López, E. y Cabero Almenara, J. (2011). Diseño y validación de un cuestionario dirigido a describir la evaluación en procesos de educación a distancia. Edutec-e, Revista Electrónica de Tecnología Educativa, 35, 126. https://doi.org/10.21556/edutec.2011.35.412

González López, I. (2005). Motivación y actitudes del alumnado universitario al inicio de la carrera. ¿Varían al egresar? Revista Electrónica de Investigación Psicoeducativa y Psicopedagógica, 3(1), 35-56. https://www.redalyc.org/pdf/2931/293121926003.pdf 
La Madriz, J. (2016). Factores que promueven la deserción del aula virtual. Orbis. Revista Científica Ciencias Humanas, 12(35), 18-40. https://www.redalyc.org/pdf/709/70948484003.pdf

Leyva López, H. P., Pérez Vera, M. G. y Pérez Vera, S. M. (2018). Google Forms en la evaluación diagnóstica como apoyo en las actividades docentes. Caso con estudiantes de la Licenciatura en Turismo. RIDE. Revista Iberoamericana para la Investigación y el Desarrollo Educativo, 9(17), 84-111. https://doi.org/10.23913/ride.v9i17.374

Lezcano, L. y Vilanova, G. (2017). Instrumentos de evaluación de aprendizaje en entornos virtuales. Perspectiva de estudiantes y aportes de docentes. Informes Científicos-Técnicos. UNPA, 9(1),1-36. https://dialnet.unirioja.es/servlet/articulo?codigo $=5919087$

Mano González, M.; Moro Cabero, M. y Melo Simões (2013). Motivaciones en la elección de la carrera universitaria: Metas y objetivos de los estudiantes de Grado en el Área de Biblioteconomía y Documentación en las Universidades de Salamanca y Coimbra. En VI Encontro Ibérico EDICIC, Porto (Portugal), 898-915. http://eprints.rclis.org/23194/

Marín-Díaz, V; Sampedro-Requena, B E.; y Vega-Gea, E. (2017). Percepciones de los estudiantes universitarios sobre las plataformas de formación. Estudio de caso. RIED, Revista Iberoamericana de Educación a Distancia, 20(1), 283-303. https://doi.org/10.5944/ried.20.1.16518

Ministerio de Educación Nacional. (2009). Deserción estudiantil en la educación superior colombiana: metodología de seguimiento, diagnóstico y elementos para su prevención. Ministerio de Educación Nacional. https://projectes.uab.cat/accedes/content/deserci\%C3\%B3n-estudiantil-en-la-educaci\%C3\%B3n-superiorcolombiana-metodolog\% $\mathrm{C} 3 \% \mathrm{ADa}$-de-seguimiento.html

Peralta Castro, R.; y Mora Rodríguez, J. (2016). El Abandono En La Educación Virtual Y A Distancia: El Caso De La Universidad Nacional Abierta Y A Distancia - UNAD. Congresos CLABES. https://revistas.utp.ac.pa/index.php/clabes/article/view/1330

Sneyers, E., y De Witte, K. (2017). The interaction between dropout, graduation rates and quality ratings in universities. Journal of the Operational Research Society, 68(4), 416-430. https://doi.org/10.1057/jors.2016.15

Soltero Avelar, R., Valenzuela Gómez, C. y Estrada de León, A. L. (2014). Un acercamiento al abandono escolar en la carrera virtual de Gestión cultural de la Universidad de Guadalajara. Revista Iberoamericana para la Investigación y el Desarrollo Educativo, 12, 1-26. Corpus ID: 177085827.

Soto, A., Briseño, M. y Gómez Zermeño, M. G. (2015). Factores de deserción de cursos virtuales para formación docente en Costa Rica. Revista Q, 10(19), 1-22. https://doi.org/10.18566/revistaq.v10n19.a03.

Tomás, J. M. y Gutiérrez, M. (2019). Aportaciones de la teoría de la autodeterminación a la predicción de la satisfacción académica en estudiantes universitarios. Revista de Investigación Educativa, 37(2), 471-485. https://doi.org/10.6018/rie.37.2.328191

Urbina Cárdenas, J. E. y Ovalle Rodríguez, G. A. (2016). Abandono y permanencia en la educación superior: Una aplicación de la teoría fundamentada. Sophia, $12(1)$ 27-37. http://www.scielo.org.co/pdf/sph/v12n1/v12n1a02.pdf

Vásquez Martínez, C. R. y Rodríguez Pérez, M. C. (2007). La deserción estudiantil en educación superior a distancia: Perspectiva teórica y factores de incidencia. Revista Latinoamericana de Estudios Educativos, 37(3-4), 107-122. https://www.redalyc.org/pdf/270/27011410005.pdf

Viale Tudela, H. E. (2014). Una aproximación teórica a la deserción estudiantil universitaria. Universidad Peruana de Ciencias Aplicadas. Revista Digital de Investigación en Docencia Universitaria, 8(1), 59-76. https://revistas.upc.edu.pe/index.php/doce. 